\title{
CT radiomics and morphological characteristics for predicting PD-L1 expression on tumor cells and tumor infiltrating lymphocytes in gastric cancer
}

\section{Xiangmei Qiao}

Department of Radiology, Nanjing Drum Tower Hospital, The Affiliated Hospital of Nanjing University Medical School

\section{Mengying Xu}

Department of Radiology, Nanjing Drum Tower Hospital, The Affiliated Hospital of Nanjing University Medical School

\section{Song Liu}

Department of Radiology, Nanjing Drum Tower Hospital, The Affiliated Hospital of Nanjing University Medical School

\section{Zhengliang Li}

State Key Lab of Novel Software Technology, Nanjing University

\section{Changfeng Ji}

Department of Radiology, Nanjing Drum Tower Hospital, The Affiliated Hospital of Nanjing University Medical School

Hui Li

Department of Radiology, Nanjing Drum Tower Hospital, The Affiliated Hospital of Nanjing University Medical School

\section{Tingting Shi}

Department of Radiology, Nanjing Drum Tower Hospital, The Affiliated Hospital of Nanjing University Medical School

\section{Lin Li}

Department of Pathology, Nanjing Drum Tower Hospital, The Affiliated Hospital of Nanjing University Medical School

\section{Qing Gu}

State Key Lab of Novel Software Technology, Nanjing University

\section{Kefeng Zhou}

Department of Radiology, Nanjing Drum Tower Hospital, The Affiliated Hospital of Nanjing University Medical School

\section{Zhengyang Zhou ( $\square$ zyzhou@nju.edu.cn)}

Department of Radiology, Nanjing Drum Tower Hospital, The Affiliated Hospital of Nanjing University Medical School https://orcid.org/0000-0003-2497-8786 


\section{Research Article}

Keywords: Stomach neoplasm, Programmed cell death ligand 1, Radiomics, Tomography, X-ray computed

Posted Date: April 30th, 2021

DOl: https://doi.org/10.21203/rs.3.rs-52520/v2

License: (c) (i) This work is licensed under a Creative Commons Attribution 4.0 International License.

Read Full License 


\section{Abstract}

Purpose To explore CT radiomics and morphological characteristics for predicting programmed cell death ligand 1 on tumor cells (PD-L1) and tumor infiltrating lymphocytes (PD-L1-TILs) status in gastric cancer (GC).

Methods From March 2019 to October 2019, 101 patients identified with GC who underwent surgery at our hospital were enrolled in this study retrospectively. Radiomic features were extracted from regions of interest manually drawn on venous CT images. Besides, 4 morphological characteristics were evaluated. The signatures based on radiomics and morphological characteristics were built using multiple classifiers (Support Vector Machine [SVM], Naive Bayes [NB], Decision Trees, and Random Forest). Receiver operating characteristic (ROC) curve was performed to assess diagnostic efficiency.

Results The adjacent adipose tissue $(p=0.009)$ and numerous radiomic features (all $p<0.05)$ differed significantly between GCs with different PD-L1 status. Six radiomic features showed significant differences between different PD-L1-TILs status (all $p<0.05$ ). The highest areas under the ROC curves (AUCs) of signatures generated by classifiers were 0.807 (SVM) and 0.729 (NB) for the prediction of PDL1 and PD-L1-TILs status, respectively.

Conclusion It was promising to predict PD-L1 status in GCs noninvasively using CT radiomics combined with morphological characteristics. It might help to improve clinical decision making with regard to immunotherapy. However, the prediction for PD-L1-TILs needs to be explored further.

\section{Introduction}

Gastric cancer (GC) is a common malignancy with the third cause of cancer-associated mortality worldwide [1]. GC is treated in a variety of ways, among which surgery is the preferred treatment. However, for unresectable locally advanced, recurrent, or metastatic GC, palliative management containing systemic treatment is recommended for patients [2].

Immunotherapy has been the focus and hotspot of clinical research since it was proposed. It includes treatments using monoclonal antibodies, cytokines, etc., which are expected to boost host anti-tumor response capability or boost the immunogenicity and sensitivity to treatment of the tumor cells $[3,4]$. Recently, the introduction of immunotherapy, particularly the utility of immune checkpoint inhibitors, has improved the prognosis of many cancers $[5,6]$. Numerous clinical works demonstrated that programmed cell death protein-1 (PD-1) (a classic immune checkpoint protein) and programmed cell death ligand 1 (PD-L1) are ideal targets for immunotherapy $[7,8]$. National Comprehensive Cancer Network guidelines for GC also comprise the treatment of positive PD-L1 expression tumors with monoclonal antibody (Pembrolizumab) [2]. What's more, Teng MW et al. showed that PD-L1 on tumor infiltrating lymphocytes (PD-L1-TILs) positive / tumor cells (PD-L1) positive pattern and PD-L1-TILs positive / PD-L1 negative pattern could be considered as "hot tumor" and benefit from PD-1 targeted therapy [9]. Thus, it is crucial to obtain the status of PD-L1 and PD-L1-TILs. 
Currently, the detection of PD-L1 and PD-L1-TILs is mainly based on endoscopic biopsy or resected specimens. Endoscopic examination is an invasive procedure with limited samples and possible selection bias, and resected specimens cannot be achieved from unresectable GCs [10]. Hence, a new method is essential to obtain the status of PD-L1 and PD-L1-TILs in GC in a simple, non-invasive, and dynamic manner. In recent years, with the increasingly extensive study of PD-L1 in tumors, medical imaging methods have been used to predict the PD-L1 expression level [11, 12]. Contrast-enhanced computed tomography (CT) is the conventional imaging modality for assessment of GC, which plays a pivotal role in staging and follow-up $[2,13]$. Nevertheless, conventional CT images mainly provide simple morphological characteristics instead of complex quantitative parameters.

Radiomics, as an emerging image analysis tool, allows extracting quantitative features noninvasively from digital medical images that enables mineable high-dimensional data to be applied in oncological practice within histological classification, lymph node metastasis, treatment response, and prognosis [1416]. As previous studies revealed, the presented radiomic-based signatures from CT and the positron emission tomography (PET)/CT were able to achieve significant and robust individualized estimation of specific PD-L1 status in non-small cell lung cancer (NSCLC) and advanced lung adenocarcinoma, respectively $[17,18]$.

With regard to the evaluation of PD-L1 and PD-L1-TILs status in GC, it has been reported that GC with positive PD-L1/PD-L1-TILs expression had elevated ${ }^{18} \mathrm{~F}$-fluorodeoxyglucose ( ${ }^{18} \mathrm{~F}$-FDG) accumulation, and ${ }^{18}$ F-FDG PET/CT had the capacity to predict the status of PD-L1/PD-L1-TILs [19]. PET/CT has higher radiation and price than $\mathrm{CT}$, which is not a routine examination. To our limited knowledge, no study has been conducted to predict PD-L1 and PD-L1-TILs expressions in GC based on CT radiomics. Moreover, there is a substantial interest in the use of machine learning algorithms for selecting optimal radiomic features from medical images and applying them to tumor evaluation, as well as in the improvement of diagnostic efficacy $[20,21]$.

Therefore, we sought to explore the capability of the signatures based on CT radiomics complement morphological characteristics to predict PD-L1 and PD-L1-TILs status in GC.

\section{Materials And Methods}

\section{Patients}

We searched patients who underwent surgery at our hospital between March 2019 and October 2019 consecutively, and 150 patients were identified with GC. The following were inclusion criteria: (1) a pathological confirmation of GC postoperatively and (2) availability of abdominal contrast-enhanced CT within 2 weeks prior to surgery. The following were exclusion criteria: (1) a history of GC treatment before surgery ( $n=4)$; (2) no definite information on PD-L1 and PD-L1-TILs $(n=2) ;(3)$ hardly visible on CT images due to the small size of the lesion ( $n=21)$; (4) insufficient distention of the stomach $(n=19)$; and (5) poor imaging quality due to respiratory or peristaltic motion $(n=3)$. The flow chart of patient selection is plotted 
in Fig. 1. Our Institutional Review Board has approved the current study, following the regulations outlined in the Declaration of Helsinki.

A total of 101 patients (male, 80; female, 21; median age, 66 years; age range, 31-86 years) conformed to the criteria.

\section{CT image acquisition}

CT examinations were performed on 64-row scanners (VCT, Discovery HD 750, GE Healthcare, and uCT 780 , United Imaging). All patients were requested to fast for at least $6 \mathrm{~h}$ and drink $600-1000 \mathrm{~mL}$ warm water to distend stomach before examination. All patients were in the supine position, and the scan covered the upper or entire abdomen. The patients were trained to hold their breath during CT scans. Following the unenhanced scan, $1.5 \mathrm{~mL} / \mathrm{kg}$ iodinated contrast agent (Omnipaque $350 \mathrm{mg} \mathrm{l} / \mathrm{mL}$, GE Healthcare) was injected intravenously at a flow rate of $3.0 \mathrm{~mL} / \mathrm{s}$ using a high-pressure syringe (Medrad Stellant CT Injector System, Medrad Inc.). Imaging was achieved with a post-injection delay of 30-40 s and $70 \mathrm{~s}$ after initiation of contrast material injection, corresponding to the arterial and venous phases, respectively. CT scan parameters: tube voltage 100-120 kV, tube current 150-250 mA, slice thickness 5 $\mathrm{mm}$, slice interval $5 \mathrm{~mm}$, field of view $35-50 \mathrm{~cm}$, matrix $512 \times 512$, rotation time $0.7 \mathrm{~s}$, and pitch 1.0875 .

\section{Image analysis}

Axial venous $\mathrm{CT}$ images of all patients were downloaded through a picture archiving and communication system and uploaded into Imaging Biomarker Explorer software. A polygonal region of interest (ROI) was manually drawn along the margin of the tumor on maximal transverse slice as illustrated in Fig. 2, carefully avoiding the normal gastric wall tissue and gastric cavity contents. ROI segmentations were performed manually by reader 1 (X.X. with 8 years of experience in abdominal imaging) who was unaware of clinicopathological information of the patients. The general location of the tumors (cardia, body, and antrum) was informed. To evaluate the interobserver reproducibility, 20 cases of CT images were randomly selected for the second ROI segmentation and feature extraction as above by reader 2 (X.X. with 8 years' experience in abdominal imaging). In total, 744 radiomic features were generated automatically from the ROls. The detailed explanations and formulas of radiomic features are displayed in supplementary material.

In addition, two radiologists evaluated the routine CT characteristics of each lesion with consensus as follows: (1) location (cardia, body, antrum, and diffuse); (2) morphological type (thickening type, mass type); (3) adjacent adipose tissue (clear, muddy); (4) lymphadenectasis (absent, present).

\section{Development and performance of signatures}

As depicted in Fig. 2, first, the intraclass correlation coefficient (ICC) was calculated to evaluate the interobserver variability of radiomic features extraction using "irr" package (vers. 0.84). Radiomic features with the ICC values $>0.8$ were regarded as highly reproducible features and initially selected. Second, the Mann-Whitney $\mathrm{U}$ test was used to select significantly different radiomic features between different PD- 
L1/PD-L1-TILs status groups, and chi-square or Fisher's exact test $(n<5)$ was used to select significantly different morphological characteristics. Third, the least absolute shrinkage and selection operator (LASSO) was used for the dimension reduction of radiomic features and morphological characteristics.

Then the optimal variables were put into our in-house software programmed with the Python Scikit-learn package (Python version 3.8, Scikit-learn version 0.22.2, http://scikit-learn.org/). The four classic algorithms including the Support Vector Machine (SVM), Naive Bayes (NB), Decision Trees (DT), and Random Forest (RF) were used to generate signatures. The ratio of the training and testing sets was 4:1. In the training phase, a popular data-preprocessing method in machine learning-Synthetic Minority Oversampling Technique was applied to handle the class imbalance problem. The models were evaluated by repeated stratified $(\mathrm{K}=5)$ cross-validation.

\section{Detection of PD-L1 andPD-L1-TILs Expression Status}

The PD-L1 and PD-L1-TILs expression status were measured through immunohistochemistry testing for paraffin-embedded tumor tissues in our study. The markers cytokeratin and the lymphocyte common antigen were used to differentiate tumor cells and tumor infiltrating lymphocytes. The positivity for PD-L1 and PD-L1-TILs was assessed by one pathologist using SP142 abcam staining. The expression for PD-L1 and PD-L1-TILs was scored according to tumor cell / tumor infiltrating lymphocyte proportion, which was defined as the percentage of tumor cells / tumor infiltrating lymphocytes with complete or partial membranous staining at any intensity.

\section{Statistical analysis}

The normality distribution of radiomic features was evaluated by the Shapiro-Wilk test. Based on the normality test results, the difference of them was analyzed by the Mann-Whitney $U$ test. Besides, the difference of morphological characteristics was assessed with the chi-square or Fisher's exact test $(n<5)$. Interobserver agreement of radiomic features was estimated with ICC (0.000-0.200: poor; 0.201-0.400: fair; 0.401-0.600: moderate; 0.601-0.800: good; 0.801-1.000: excellent). Receiver operating characteristic (ROC) analysis and the area under the ROC curve (AUC) were performed to evaluate the diagnostic performance of signatures. Precision, recall, and F1 score were calculated to assess the machine learning models. Precision is defined as true-positive results divided by the sum of false-positive and true-positive results. Recall is defined as true-positive results divided by the sum of true-positive and false-negative results. F1 score is defined as the harmonic mean of precision and recall. All those statistical analyses were performed with SPSS (version 22.0 for Microsoft Windows x64, SPSS), MedCalc Statistical Software (version 11.4.2.0 MedCalc Software bvba; http://www.medcalc.org; 2011), R software package (version 3.5.2: http:// www.Rproject.org), and Python Scikit-learn package (Python version 3.8, Scikit-learn version 0.22 .2 , http://scikit-learn.org/). A two-tailed $p$ value $<0.05$ was considered statistically significant.

\section{Results}

\section{Qualitative Data Analysis}


The demographic data and pathological information of the included patients are summarized in Table 1. As shown in Table 2, the adjacent adipose tissue showed a significant difference between negative and positive PD-L1 expressions in GCs $(p=0.009)$. In addition, there were no significant differences in location, morphological type, adjacent adipose tissue, and lymphadenectasis between GCs with different PD-L1TILs status (Table 2).

\section{Quantitative Data Analysis}

After assessing the reproducibility, the data derived from the tumor ROI on venous $\mathrm{CT}$ images were reduced to 650 robust features (interobserver ICC values $>0.8$ ) for the subsequent analysis.

There were significant differences in terms of 332 radiomic features derived from venous CT images between negative and positive PD-L1 expressions GCs. The diagnostic performance of those features ranged from 0.646 to 0.756 . Besides, six features differed significantly between negative and positive PDL1-TILs expressions GCs. The AUCs ranged from 0.665 to 0.668 .

\section{Diagnostic Performance of Signatures}

All the significantly different variables were placed into LASSO for dimension reduction. Ultimately, 11 (adjacent adipose tissue, F2135-4ClusterTendendcy, F2315-4ClusterTendendcy, F290-7Homogeneity, F290-4InformationMeasureCorr2, F2270-7Homogeneity, F3 90LongRunLowGrayLevelEmpha, F204SumEntropy, F2180-4SumEntropy, F2135-1InverseVariance, and F2315-1InverseVariance) and 4 (F21357Homogeneity, F2315-7Homogeneity, F2135-7Homogeneity2, and F2135-7MaxProbability) optimal variables were reserved to develop the models of PD-L1 and PD-L1-TILs, respectively.

The diagnostic efficacy of signatures generated by machine learning algorithms was various. The signature based on SVM showed the best performance in predicting the PD-L1 status of GCs with an AUC of 0.807 (Table 3). With respect to PD-L1-TILs, the signature created by NB was superior to the others with an AUC of 0.729 (Table 4). The models established by DT and RF for PD-L1-TILs status did not achieve high enough diagnostic efficiency (Table 4).

\section{Discussion}

PD-1 is a classic immune checkpoint protein, and the utility of PD-1 targeted therapy (depending on the status of PD-L1 and PD-L1-TILs) is beneficial to improve the prognosis of many cancers [3]. In this study, we extracted $744 \mathrm{CT}$ radiomic features and 4 morphological characteristics of GCs. Plenty of radiomic features and adjacent adipose tissue differed significantly between GCs with different PD-L1 status, and several radiomic features showed significant differences between GCs with different PD-L1-TILs status. After the feature selection, these signatures based on classifiers (SVM, NB, DT, and RF) were competent to predict the status of PD-L1 and PD-L1-TILs in GCs.

Nowadays, CT radiomics has been used widely in tumor assessment [14-16], yet only a few of them focused on PD-L1. Our study found that there were abundant radiomic features with statistical 
significance between GCs with different PD-L1 expressions, whereas the diagnostic performance was not efficient enough with AUCs ranging from 0.646 to 0.756 . All those features adding significant morphological characteristics were placed into LASSO for dimension reduction, and after using multiple classifiers, the diagnostic efficiency of SVM, NB, and RF increased except DT with AUCs of $0.807,0.779$, and 0.774 , respectively. It indicated that the SVM might be a more suitable algorithm for evaluating the PD-L1 expression in GC. To our limited knowledge, no previous study focused on CT radiomics to predict PD-L1 status of GC, but Chen R, et al. applied SUVmax based on PET to investigate PD-L1 expression in GC with an AUC of 0.822 [19]. Although the AUC was slightly higher than ours, the sample size was relatively small. Besides, for some patients, the specimens for the detection of PDL-1 were obtained only by endoscopic biopsy.

Meanwhile, the evaluation of PD-L1-TILs has also attracted attention. In a study by Teng MW et al., the status of PD-L1-TILs had an influence on promoting suitable clinical decision-making, as did PD-L1 status [9]. Two recent works $[19,25]$ have demonstrated that SUVmax based on PET had the potential to be an imaging biomarker for the prediction of PD-L1-TILs expression in NSCLC and GC but with unsatisfactory diagnostic efficacy. In this study, we attempted to apply CT radiomics analysis to predict the PD-L1-TILs status of GC, and several parameters showed significant differences. The diagnostic efficiency was not high enough even operating the classifiers. In this scenario, an effective predictive method over the PD-L1-TILs status deserves further exploration.

Although quantitative analysis has developed rapidly, there are still continuous reports $[22,23]$ on the morphological characteristics participating in the tumor evaluation due to their visualization and conventional use. Hence, we also evaluated the morphological characteristics and found that muddy adjacent adipose tissue was more common in positive PD-L1 expression GC. The positive PD-L1 expression tumor immune response may be stronger, which has the potential to result in edema of lymphatic vessels in the adipose tissue around the lesion, but this needs pathological confirmation further. Depending on our previous literature investigation, no study on the prediction of PD-L1 status in GC by CT morphological characteristics was found. While in lung adenocarcinoma, Gouji, et al. [26] revealed that PD-L1 status was significantly associated with the presence of convergence, surrounding ground-glass opacity, notching, speculation, and cavitation (all $p<0.01$ ). In addition, we also explored the relationship between CT morphological characteristics and PD-L1-TILs status. Unfortunately, no significant difference was observed.

Certain limitations of our study deserve consideration. First, it was a single centre retrospective study with a relatively small sample size. Prospective multicentre studies with large samples need to be carried out to confirm our results further. Second, our feature extraction was restricted to lesions on venous phase, because a prior study suggested [26] that radiomic features derived from venous phase images had superior discrimination of tumor tissue from adjacent normal gastrointestinal wall. Third, ROls were delineated on the maximal slice images since tumor on that section is the clearest, which might not reflect the overall information of the tumor. 
In conclusion, it was promising to predict PD-L1 status in GCs noninvasively using CT radiomics combined with morphological characteristics. It might help to improve clinical decision making with regard to immunotherapy. However, the prediction for PD-L1-TILs needs to be explored further.

\section{Declarations}

\section{Data availability}

Data are available upon request to the corresponding author.

\section{Compliance with Ethical Standards}

\section{Conflict of interest}

The authors of this manuscript declare no relevant conflicts of interest, and no relationships with any companies, whose products or services may be related to the subject matter of the article.

\section{Ethical approval}

Institutional review board approval was obtained.

\section{Informed consent}

Written informed consent was waived.

\section{Reference}

1. Bray F, Ferlay J, Soerjomataram I, Siegel RL, Torre LA, Jemal A (2018) Global cancer statistics 2018: GLOBOCAN estimates of incidence and mortality worldwide for 36 cancers in 185 countries. CA Cancer J Clin 68(6):394-424.

2. Ajani JA, D'Amico TA, Almhanna K, Bentrem DJ, Chao J, Das P et al. National Comprehensive Cancer Network. NCCN Clinical Practice Guidelines in Oncology. Gastric Cancer, Version 1.2019. Available at: https://www.nccn.org/professionals/physician_gls/PDF/gastric.pdf. Accessed 14 May 2019.

3. Lazăr DC, Avram MF, Romoșan I, Cornianu M, Tăban S, Goldiș A (2018) Prognostic significance of tumor immune microenvironment and immunotherapy: Novel insights and future perspectives in gastric cancer. World J Gastroenterol 24(32):3583-3616.

4. Niccolai E, Taddei A, Prisco D, Amedei A (2015) Gastric cancer and the epoch of immunotherapy approaches. World J Gastroenterol 21(19):5778-5793.

5. Robert C, Thomas L, Bondarenko I, et al (2011) Ipilimumab plus dacarbazine for previously untreated metastatic melanoma. N Engl J Med 364(26):2517-2526.

6. Bellmunt J, Powles T, Vogelzang NJ (2017) A review on the evolution of PD-1/PD-L1 immunotherapy for bladder cancer: The future is now. Cancer Treat Rev 54:58-67. 
7. Philips GK, Atkins M (2015) Therapeutic uses of anti-PD-1 and anti-PD-L1 antibodies. Int Immunol 27(1):39-46.

8. Muro K, Chung HC, Shankaran V, et al (2016) Pembrolizumab for patients with PD-L1-positive advanced gastric cancer (KEYNOTE-012): a multicentre, open-label, phase 1b trial. Lancet Oncol 17(6):717-726.

9. Teng MW, Ngiow SF, Ribas A, Smyth MJ (2015) Classifying Cancers Based on T-cell Infiltration and PD-L1. Cancer Res 75(11):2139-2145.

10. Lee IS, Park YS, Lee JH, et al (2013) Pathologic discordance of differentiation between endoscopic biopsy and postoperative specimen in mucosal gastric adenocarcinomas. Ann Surg Oncol 20(13):4231-4237.

11. Wu T, Zhou F, Soodeen-Lalloo AK, et al (2019) The Association Between Imaging Features of TSCT and the Expression of PD-L1 in Patients With Surgical Resection of Lung Adenocarcinoma. Clin Lung Cancer 20(2):e195-e207.

12. Zhao L, Zhuang Y, Fu K, et al (2020) Usefulness of [18F]fluorodeoxyglucose PET/CT for evaluating the PD-L1 status in nasopharyngeal carcinoma. Eur J Nucl Med Mol Imaging 47(5):1065-1074.

13. Chen CY, Hsu JS, Wu DC, et al (2007) Gastric cancer: preoperative local staging with 3D multidetector row CT--correlation with surgical and histopathologic results. Radiology 242(2):472-482.

14. Li Q, Qi L, Feng QX, et al (2019) Machine Learning-Based Computational Models Derived From LargeScale Radiographic-Radiomic Images Can Help Predict Adverse Histopathological Status of Gastric Cancer. Clin Transl Gastroenterol 10(10):e00079.

15. Jin X, Zheng X, Chen $D$, et al (2019) Prediction of response after chemoradiation for esophageal cancer using a combination of dosimetry and CT radiomics. Eur Radiol 29(11):6080-6088.

16. Ji GW, Zhang YD, Zhang H, et al (2019) Biliary Tract Cancer at CT: A Radiomics-based Model to Predict Lymph Node Metastasis and Survival Outcomes. Radiology 290(1):90-98.

17. Jiang M, Sun D, Guo Y, et al (2020) Assessing PD-L1 Expression Level by Radiomic Features From PET/CT in Nonsmall Cell Lung Cancer Patients: An Initial Result. Acad Radiol 27(2):171-179.

18. Yoon J, Suh YJ, Han K, et al (2020) Utility of CT radiomics for prediction of PD-L1 expression in advanced lung adenocarcinomas. Thorac Cancer 11(4):993-1004.

19. Chen R, Chen Y, Huang G, Liu J (2019) Relationship between PD-L1 expression and 18F-FDG uptake in gastric cancer. Aging (Albany NY) 11(24):12270-12277.

20. Song L, Zhu Z, Mao L, et al (2020) Clinical, Conventional CT and Radiomic Feature-Based Machine Learning Models for Predicting ALK Rearrangement Status in Lung Adenocarcinoma Patients. Front Oncol 10:369.

21. Feng Z, Zhang L, Qi Z, Shen Q, Hu Z, Chen F (2020) Identifying BAP1 Mutations in Clear-Cell Renal Cell Carcinoma by CT Radiomics: Preliminary Findings. Front Oncol 10:279.

22. Hu B, Chen W, Zhang Y, Shi H, Cheng D, Xiu Y (2020) 18F-FDG maximum standard uptake value predicts PD-L1 expression on tumor cells or tumor-infiltrating immune cells in non-small cell lung 
cancer. Ann Nucl Med 34(5):322-328.

23. Gao F, Li M, Zhang Z, et al (2019) Morphological classification of pre-invasive lesions and early-stage lung adenocarcinoma based on CT images. Eur Radiol 29(10):5423-5430.

24. Tu W, Sun G, Fan L, et al (2019) Radiomics signature: A potential and incremental predictor for EGFR mutation status in NSCLC patients, comparison with CT morphology. Lung Cancer 132:28-35.

25. Toyokawa G, Takada K, Okamoto T, et al (2017) Computed Tomography Features of Lung Adenocarcinomas With Programmed Death Ligand 1 Expression. Clin Lung Cancer 18(6):e375-e383.

26. Huang YQ, Liang CH, He L, et al (2016) Development and Validation of a Radiomics Nomogram for Preoperative Prediction of Lymph Node Metastasis in Colorectal Cancer. J Clin Oncol 34(18):21572164.

\section{Tables}

Table 1 Demographic data and pathological information in patients with GC

\begin{tabular}{|c|c|c|c|c|}
\hline \multirow[t]{2}{*}{ Characteristics } & \multicolumn{2}{|c|}{ PD-L1 } & \multicolumn{2}{|c|}{ PD-L1-TIL } \\
\hline & Negative (\%) & Positive (\%) & Negative (\%) & Positive (\%) \\
\hline \multicolumn{5}{|l|}{ Gender } \\
\hline Male & $66(81.5)$ & $14(70.0)$ & $10(71.4)$ & $70(80.5)$ \\
\hline Female & $15(18.5)$ & $6(30.0)$ & $4(28.6)$ & $17(19.5)$ \\
\hline \multicolumn{5}{|l|}{ Age (y) } \\
\hline$\leq 60$ & $24(29.6)$ & $9(45.0)$ & $6(42.9)$ & $27(31.0)$ \\
\hline$>60$ & $57(70.4)$ & $11(55.0)$ & $8(57.1)$ & $60(69.0)$ \\
\hline \multicolumn{5}{|l|}{ T stage } \\
\hline 1 & $3(3.7)$ & $0(0.0)$ & $0(0.0)$ & $3(3.4)$ \\
\hline 2 & $12(14.8)$ & $4(20.0)$ & $2(14.3)$ & $14(16.1)$ \\
\hline 3 & $48(59.3)$ & $5(25.0)$ & $10(71.4)$ & $43(49.4)$ \\
\hline 4 & $18(22.2)$ & $11(55.0)$ & $2(14.3)$ & $27(31.0)$ \\
\hline \multicolumn{5}{|l|}{$\mathrm{N}$ stage } \\
\hline 0 & $23(28.4)$ & $1(5.0)$ & $3(21.4)$ & $66(75.9)$ \\
\hline $1-3$ & $58(71.6)$ & $19(95.0)$ & $11(78.6)$ & $21(24.1)$ \\
\hline \multicolumn{5}{|c|}{ Lymphovascular invasion } \\
\hline Negative & $33(40.7)$ & $4(20.0)$ & $6(42.9)$ & $31(35.6)$ \\
\hline Positive & $48(59.3)$ & $16(80.0)$ & $8(57.1)$ & $56(64.4)$ \\
\hline \multicolumn{5}{|l|}{ Neural invasion } \\
\hline Negative & $20(24.7)$ & $1(5.0)$ & $3(21.4)$ & $18(20.7)$ \\
\hline Positive & $61(75.3)$ & $19(95.0)$ & $11(78.6)$ & $69(79.3)$ \\
\hline
\end{tabular}

$* p<0.05$ with chi-square test or Fisher's exact test $(\mathrm{n}<5)$.

GC, gastric cancer; PD-L1, programmed cell death ligand 1 on tumor cell; PD-L1-TIL, programmed cell death ligand 1 on tumor infiltrating lymphocyte.

Table 2 Univariate analysis of CT characteristics in GCs 


\begin{tabular}{|c|c|c|c|c|c|c|}
\hline \multirow[t]{2}{*}{ haracteristics } & \multicolumn{3}{|c|}{ PD-L1 } & \multicolumn{3}{|c|}{ PD-L1-TIL } \\
\hline & negative (\%) & positive (\%) & ) $p$ & negative (\%) & positive (\%) & $p$ \\
\hline socation & & & 0.320 & & & 0.615 \\
\hline Cardia & $33(40.7)$ & $5(25.0)$ & & $5(35.7)$ & $33(37.9)$ & \\
\hline Body & 15 (18.5) & $7(35.0)$ & & $2(14.3)$ & $20(23.0)$ & \\
\hline Antrum & $30(37.0)$ & $8(40.0)$ & & $6(42.9)$ & $32(36.8)$ & \\
\hline Diffuse & $3(3.7)$ & $0(0.0)$ & & $1(7.1)$ & $2(2.3)$ & \\
\hline Uorphologic type & & & 0.596 & & & 0.592 \\
\hline Thickening type & $75(92.6) \quad 2$ & $20(100.0)$ & & $14(100.0)$ & $81(93.1)$ & \\
\hline Mass type & $6(7.4)$ & $0(0.0)$ & & $0(0.0)$ & $6(6.9)$ & \\
\hline Idjacent adipose tissue & & & $.009 *$ & & & 0.105 \\
\hline Clear & $64(79.0)$ & $10(50.0)$ & & $13(92.9)$ & $61(70.1)$ & \\
\hline Muddy & $17(21.0)$ & $10(50.0)$ & & $1(7.1)$ & $26(29.9)$ & \\
\hline .ymphadenectasis & & & 0.133 & & & 0.729 \\
\hline Absent & $66(81.5)$ & $13(65.0)$ & & $12(85.7)$ & $67(77.0)$ & \\
\hline Present & 15 (18.5) & 7 (35.0) & & $2(14.3)$ & $20(23.0)$ & \\
\hline
\end{tabular}

${ }^{*} p<0.05$ with chi-square test or Fisher's exact test $(\mathrm{n}<5)$.

CT, computed tomography; GC, gastric cancer; PD-L1, programmed cell death ligand 1 on tumor cell; PD-L1-TIL, programmed cell death ligand 1 on tumor infiltrating lymphocyte.

Table 3 The diagnostic efficiency of signatures based on classifiers in GCs with different PD-L1 expressions

\begin{tabular}{llllll}
\hline Classifiers & Accuracy & Precision & Recall & F1 & AUC \\
\hline Support Vector Machine & 0.601 & 0.359 & 0.900 & 0.496 & 0.807 \\
Naive Bayes & 0.651 & 0.384 & 0.900 & 0.523 & 0.779 \\
Decision Trees & 0.631 & 0.456 & 0.800 & 0.460 & 0.698 \\
Random Forest & 0.671 & 0.472 & 0.850 & 0.531 & 0.774 \\
\hline
\end{tabular}

GC, gastric cancer; PD-L1, programmed cell death ligand 1 on tumor cell; AUC, area under the receiver operating characteristic curve.

Table 4 The diagnostic efficacy of signatures based on classifiers in GCs with different PD-L1-TIL expressions

\begin{tabular}{llllll}
\hline Classifiers & Accuracy & Precision & Recall & F1 & AUC \\
\hline Support Vector Machine & 0.811 & 0.870 & 0.920 & 0.893 & 0.657 \\
Naive Bayes & 0.735 & 0.890 & 0.784 & 0.825 & 0.729 \\
Decision Trees & 0.624 & 0.889 & 0.644 & 0.742 & 0.549 \\
Random Forest & 0.623 & 0.911 & 0.633 & 0.737 & 0.501 \\
\hline
\end{tabular}


GC, gastric cancer; PD-L1-TIL, programmed cell death ligand 1 on tumor infiltrating lymphocyte; AUC, area under the receiver operating characteristic curve.

\section{Figures}

Patients underwent surgery at our hospital between March 2019 and October 2019 identified with GC $(n=150)$

A history of GC treatment before surgery $(n=4)$

Hardly visible on CT images due to the small size of the lesion $(n=21)$

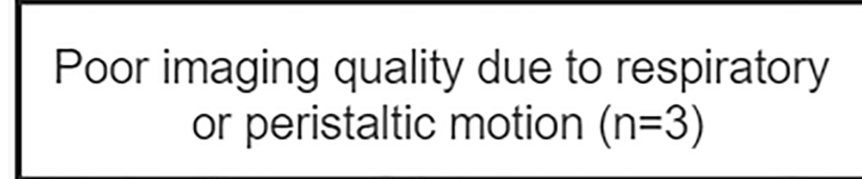

Insufficient distention of the stomach $(n=19)$

No definite information on PD-L1 and PD-L1-TILs $(n=2)$
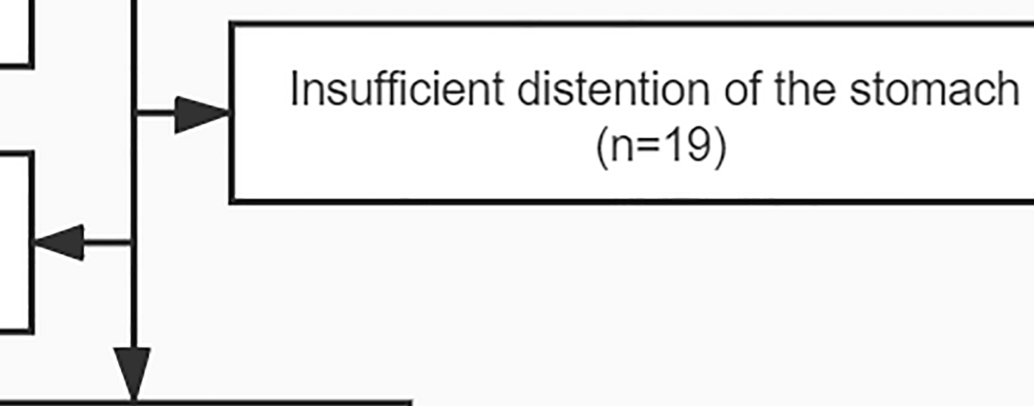

\section{Enrolled patients} $(n=101)$

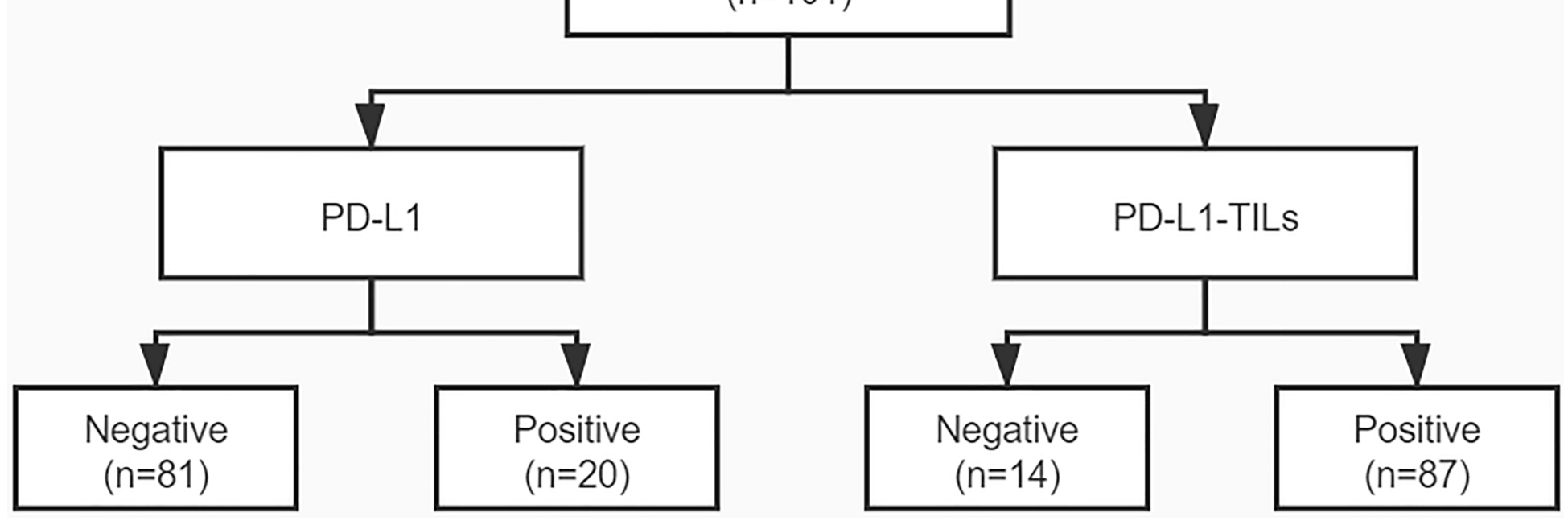

\section{Figure 1}

The flow chart of the enrolled patients in our study. GC, gastric cancer; CT, computed tomography; PD-L1, programmed cell death ligand 1 on tumor cell; PD-L1-TIL, programmed cell death ligand 1 on tumor 
infiltrating lymphocyte.

a

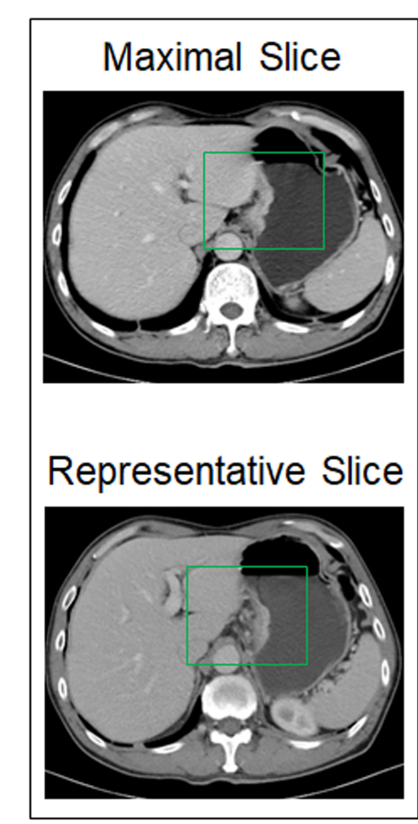

b Feature Extraction
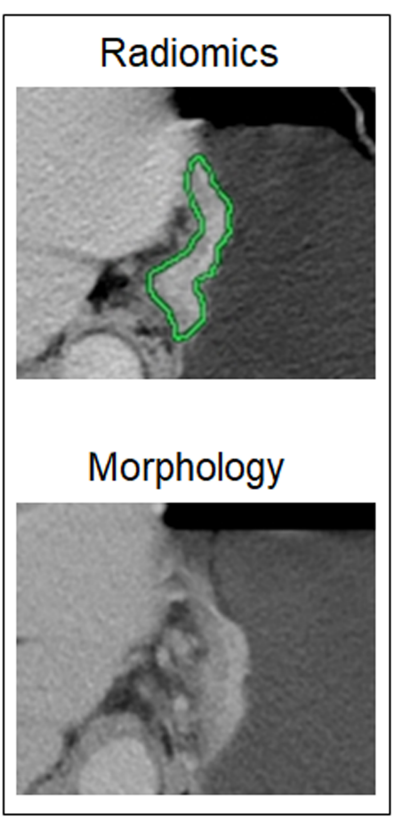

c Signatures Building

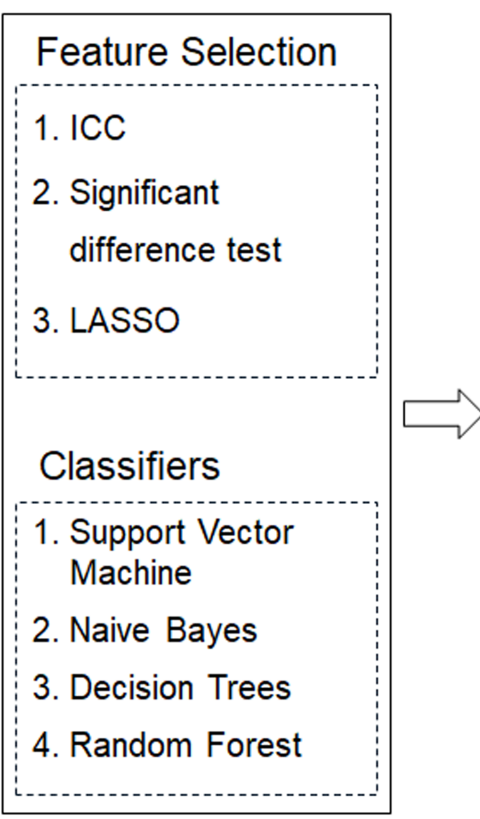

d Prediction

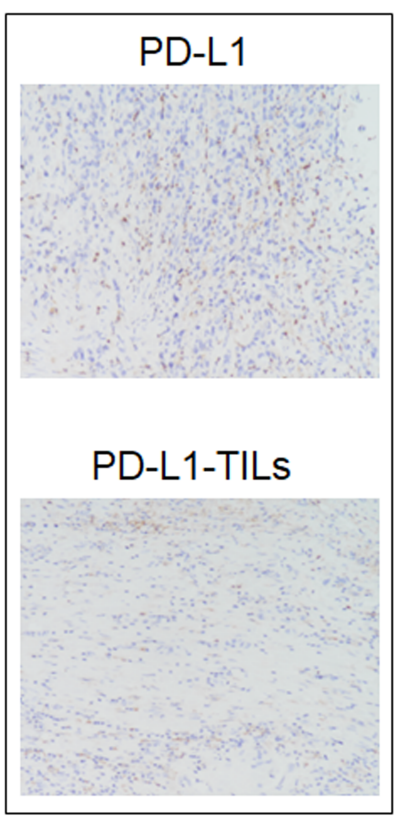

Figure 2

The workflow of this study. On venous CT images, the maximal transverse and representative slices of the tumor (a) were selected to extract radiomic features and morphological characteristics, respectively. An ROI was delineated manually along the border of the tumor and zoomed in $300 \%$ (b). c ICC, significant difference test, and LASSO were used to screen optimal radiomic features and morphological characteristics. Signatures based on classifiers were constructed using selected radiomic features and morphological characteristics. d To predict PD-L1 and PD-L1-TILs expressions in gastric cancer. CT, computed tomography. ROI, region of interest; ICC, intraclass correlation coefficient; LASSO, least absolute shrinkage and selection operator; PD-L1, programmed cell death ligand 1 on tumor cells; PD-L1TILs, programmed cell death ligand 1 on tumor infiltrating lymphocytes.

\section{Supplementary Files}

This is a list of supplementary files associated with this preprint. Click to download.

- SupplementaryMaterial.docx 